\title{
A MATCHING METHOD TO REDUCE THE INFLUENCE OF SAR GEOMETRIC DEFORMATION
}

\author{
Chao Gao ${ }^{1,2}$, Guochao Xue ${ }^{1,2}$ \\ ${ }^{1}$ College of Geomatics, Shandong University of Science and Technology, Qingdao,266590, China, gc910909@163.com. \\ ${ }^{2}$ Chinese Academy of Surveying and Mapping, Beijing 100830, China
}

Keywords: SAR image, image matching, SURF, SNCC, geometrical deformations

\begin{abstract}
:
There are large geometrical deformations in SAR image, including foreshortening, layover, shade, which leads to SAR Image matching with low accuracy . Especially in complex terrain area, the control points are difficult to obtain, and the matching is difficult to achieve. Considering the impact of geometric distortions in SAR image pairs, a matching algorithm with a combination of speeded up robust features (SURF) and summed of normalize cross correlation (SNCC) was proposed, which can avoid the influence of SAR geometric deformation. Firstly, SURF algorithm was utilized to predict the search area. Then the matching point pairs was selected based on summed of normalized cross correlation. Finally, false match points were eliminated by the bidirectional consistency. SURF algorithm can control the range of matching points, and the matching points extracted from the deformation area are eliminated, and the matching points with stable and even distribution are obtained. The experimental results demonstrated that the proposed algorithm had high precision, and can effectively avoid the effect of geometric distortion on SAR image matching. Meet accuracy requirements of the block adjustment with sparse control points.
\end{abstract}

\section{INTRODUCTION}

The SAR sensor has side-view imaging method, resulting in the difference of SAR image deformation Large, there is a shadow, overlapping cover and other phenomena, but also the lack of texture information in the mountain area, increasing the SAR image matching difficulty. However, SAR image matching is an essential part of SAR data processing and application.

In this paper, I adopt the combination of feature point extraction and summed normalized cross correlation (SNCC) to obtain evenly distributed matching points, and eliminates the error point pairs based on the two-way consistency constraint to improve the matching accuracy.

\section{FEATURE POINT DETECTION BASE ON SURF}

\subsection{Integral image}

The primary workhorse of SURF algorithm is based on the integral image for speed up. So that, the first work we need to do is to get the integral image. The establishment of the integral image greatly reduces the computation time. The value of any point $(i, j)$ in the integral image is the sum of the pixel values of the corresponding diagonal region from the upper left corner of the original image to any point (i, j), as formula (1):

$$
\mathrm{f}(\mathrm{i}, \mathrm{j})=\sum_{i^{\prime} \leq i, j^{\prime} \leq j} p\left(i^{\prime}, j^{\prime}\right)
$$

To calculate the sum of the pixels in any of the rectangular regions of an image, simply calculate the value of the four vertices of the rectangular region in the integral image. Thus, SURF algorithm operation does not change with the size of the image to change the operation time.

\subsection{Interest point detection}

The SURF detector is based on the Hessian matrix which also belongs to Blobs detector. The Hessian matrix in x at scale $\sigma$ is defined as follows:

$$
\mathrm{H}(\mathrm{x}, \sigma)=\left(\begin{array}{ll}
l_{x x}(x, \sigma) & l_{x y}(x, \sigma) \\
l_{x y}(x, \sigma) & l_{y y}(x, \sigma)
\end{array}\right)
$$

In order to reduce the calculating complexity of the algorithm, we approximate second order Gaussian derivatives with box filers. Then Image convolutions with these box filters can be computed rapidly only by using integral image. By changing the size of the filter box to form a multi-scale spatial function and the original image in different directions for convolution calculation, We denote them by $D_{x x}, D_{x y}, D_{y y}$ instead of $l_{x x}, l_{x y}, l_{y y}$, So the Hessian matrix can be simplified to Formula III:

$$
\operatorname{Det}(\text { Hessian })=D_{x x} D_{y y}-\left(\omega D_{x x}\right)
$$

where $\quad \omega=0.9$

The SURF algorithm uses integral images and box filters, eliminating the need for sampling operations and building scale space directly by constantly changing the size of the box filter. Then non-maximal suppression is performed in the scale space to find the local extreme point (Hessian determinant). Finally, the scale and the image space are interpolated to obtain the exact location and scale information of the feature points.

\subsection{Interest Point Descriptor}

We compute the main orientation around the interest point. For one thing, Construct a circular region of $6 \mathrm{~s}(\mathrm{~s}$ is the scale at which the interest point was detected) radius around the detected interest points which is aim to assign a reproducible orientation. Then, calculate the Haarwavelet responses in the $\mathrm{x}$, and $\mathrm{y}$ 
direction within this circular. Take the feature point as the center and make a sliding search with a sector whose central angle is $60^{\circ}$ as shown in the figure 1 , and calculate the sum of the Harr wavelet responses in the fan-shaped area. The resulting maximum is then chosen to describe the orientation of the interest point descriptor.

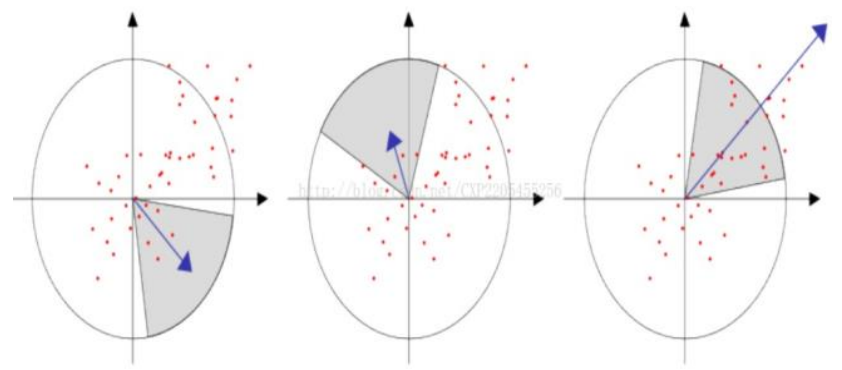

Figure 1. Interest Point Descriptor

Interest point descriptors are constructed by extracting square windows around the interest points. The windows are split up into $4 \times 4$ square sub-regions with $5 \times 5$ regularly spaced sample points inside. In each sub-region, we compute the Haar wavelet responses in horizontal and vertical directions ( $\mathrm{dx}$ and dy ) and the responses are weighted by a Gaussian centered on the interest point. Where after, the wavelet responses along $\mathrm{x}$ and the responses along y are summed up over each sub-region. Furthermore, the absolute values of responses in both $\mathrm{x}$ and $\mathrm{y}$ directions are summed in order to obtain information about the polarity of the image intensity changes. Consequently, the underlying intensity pattern of each sub-region is described by a vector

$$
v=\left(\sum d_{x}, \sum d_{y}, \sum\left|d_{x}\right|, \sum\left|d_{y}\right|\right)
$$

\section{SUMMED NORMALIZED CROSS CORRELATION}

\subsection{NCC}

The normalized cross-correlation (NCC) is optimal for compensating Gaussian noise and the correlation values are constrained to the interval of $[-1,1]$, which eases the selection of a threshold for rejecting bad matches. The main disadvantage of NCC is the strong fattening effect compared to other matching costs. For two patches from the two camera images left and right the normalized cross-correlation (NCC) is defined:

$\mathrm{NCC}=\frac{\sum_{i=1}^{n}\left(x_{i}-\bar{x}\right)\left(y_{i}-\bar{y}\right)}{\sqrt{\sum_{i=1}^{n}\left(x_{i}-\bar{x}\right)^{2} \sum_{i=1}^{n}\left(y_{i}-\bar{y}\right)^{2}}}=\frac{1}{n} \sum_{i=1}^{n} x_{i}^{\prime} y_{i}^{\prime}$

where $\left\{\begin{array}{l}x_{i}^{\prime}=\frac{\left(x_{i}-\bar{x}\right)}{\sigma_{x}} \\ y_{i}^{\prime}=\frac{\left(y_{i}-\bar{y}\right)}{\sigma_{y}}\end{array}\right.$

$x_{i}, y_{i}=$ the pixel position of the anchor point $\sigma_{x}, \sigma_{y}=$ standard deviation

$\mathrm{n}=$ the number of pixels in the matching window

\subsection{SNCC}

SNCC is a matching method developed on the basis of NCC. It does not directly calculate the normalized correlation coefficient between matching windows. Instead, it sets a large matching window, as shown by the black rectangle in the figure, and then sets a smaller sliding match window, as shown by the red rectangle in Fig 2. Then, for each pixel in the larger matching window, use the small matching window to calculate the NCC value according to formula (5), and then average the $\mathrm{NCC}$ values of all the pixels in the large matching window. So the SNCC is defined:

$$
\mathrm{SNCC}=\frac{1}{n} \sum_{i=1}^{n} N C C_{i}
$$

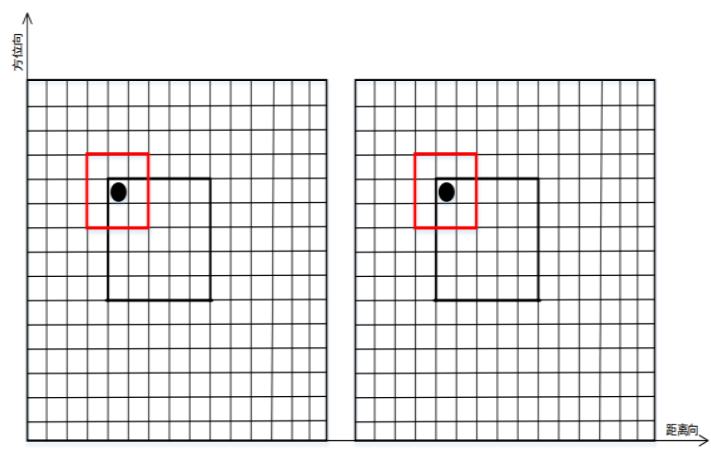

Figure 2. SNCC calculation diagram

\subsection{The Bidirectional Consistency}

SAR images usually have large geometrical deformation, there is an error in the matching result of SNCC. In order to improve the matching accuracy, bidirectional consistency selection is adopted to eliminate false matching points. Assuming that the point to be matched $p_{i}$ is forward matched, the point with the same name obtained is $p_{i}^{\prime}$; then $p_{i}^{\prime}$ is the point to be matched and the matching is performed in the reverse direction to obtain the point with the same name as $p_{i}$.

\section{RESULTS\&ANALYSIS}

\subsection{Experimental data and results}

To validate the validity of the algorithm in SAR image matching, we select two sets of SAR data, Used VC++, OpenCV and GDAL programming as software platform. We used two SAR images from COSMO for our SURF experiment, this two images (as show in Fig3), the size of images (a) is $401 \times 501$ pixels, detected in 325 interest points in the left part of (a) and 355 interest points in the right part of (a). (b) is $401 \times 435$ pixels, detected in 171 interest points in the left part of (b) and 224 interest points in the right part of (b). Final results of the match as shown in table1.

\begin{tabular}{|c|c|c|c|c|c|}
\hline $\begin{array}{c}\text { SAR } \\
\text { image }\end{array}$ & $\begin{array}{c}\text { Interest } \\
\text { points of } \\
\text { Reference } \\
\text { image }\end{array}$ & $\begin{array}{c}\text { Interest } \\
\text { points } \\
\text { of Test } \\
\text { image }\end{array}$ & $\begin{array}{c}\text { Match } \\
\text { points }\end{array}$ & Time(s) & Accuracy \\
\hline 1 & 225 & 255 & 123 & 2.1 & $54.6 \%$ \\
\hline 2 & 171 & 224 & 80 & 1.3 & $46.7 \%$ \\
\hline
\end{tabular}

Table1. Test results with SURF 

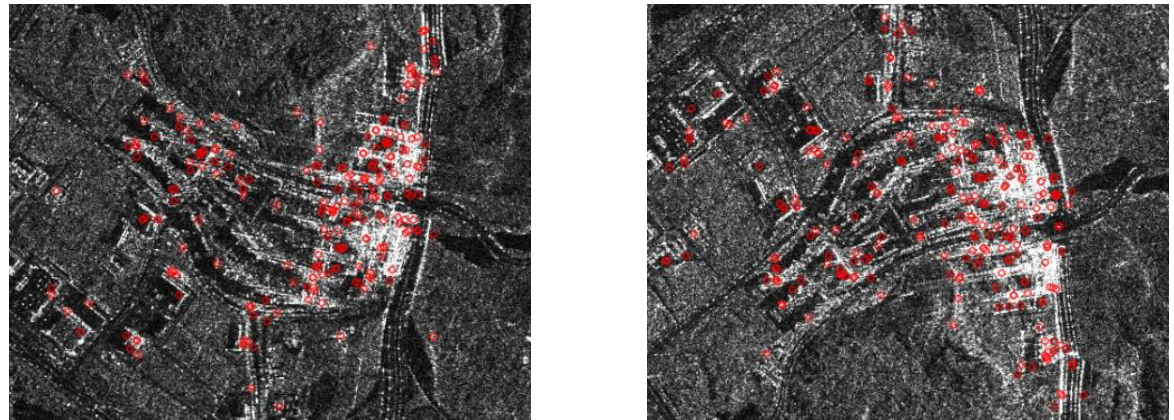

(a) Test results with SURF, Some interest points are uneven distribution and in the deformed area
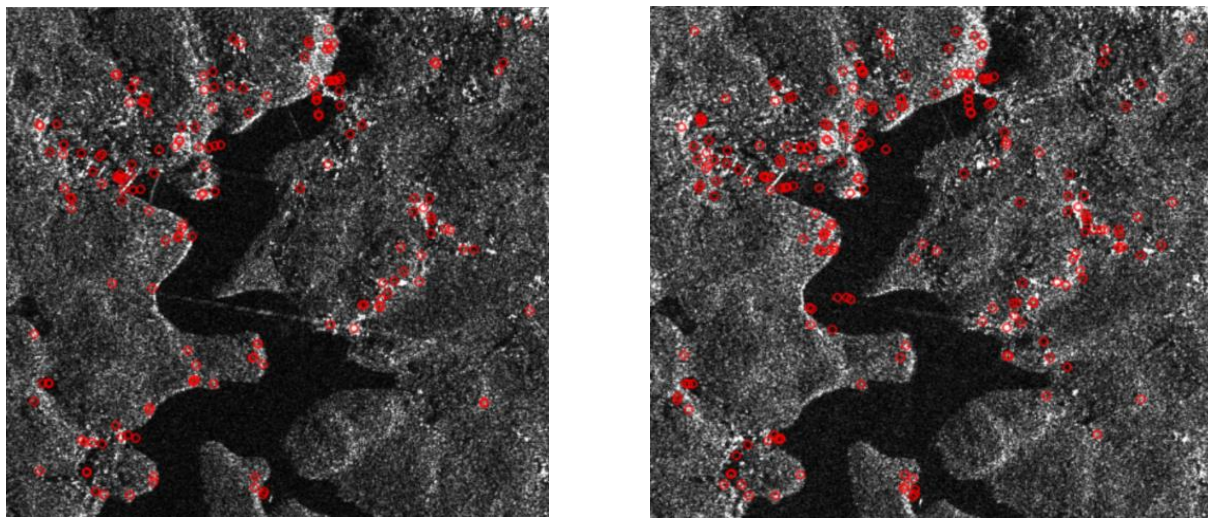

(b) Test results with SURF, Some interest points in the water

Figure 3. The result of SURF

Table 1 shows more detail test results of SURF. A large number of interest points are detected. When SURF in the use of RANSAC to remove the wrong match point. The accuracy of match is about $50 \%$. But from the Fig 1 it is obvious that some match points in the deformed area or in the water. These points will affect the actual mapping. In this paper, we chose the interest points by SURF as the search area, then used SNCC to extract matching points, The matching center is the matching point. In the SNCC algorithm for the S SAR image, larger match window set to $45 \times 45$, smaller match window set to $9 \times 9$, the results as show in table 2. Final results of the match as shown in Fig 4 and Fig5.

\begin{tabular}{|c|c|c|c|c|}
\hline $\begin{array}{c}\text { SAR } \\
\text { image }\end{array}$ & $\begin{array}{c}\text { Interest } \\
\text { points } \\
\text { with } \\
\text { SURF }\end{array}$ & $\begin{array}{c}\text { matching } \\
\text { points } \\
\text { with our } \\
\text { method }\end{array}$ & $\begin{array}{c}\text { Evaluation of } \\
\text { position } \\
\text { accuracy/pixel }\end{array}$ & Accuracy \\
\hline 1 & 30 & 28 & 0 & $93.3 \%$ \\
\hline 2 & 30 & 27 & 1 & $90.0 \%$ \\
\hline
\end{tabular}

Table 2. Test results of SAR image with our method
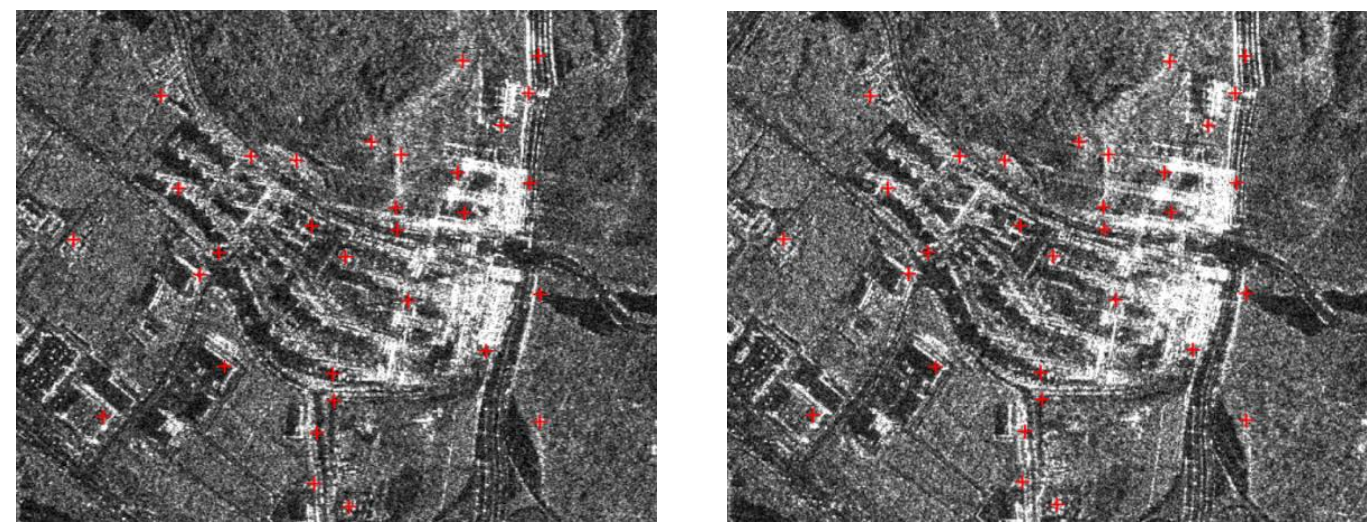

Figure4. Results of Test Image in Building Area 

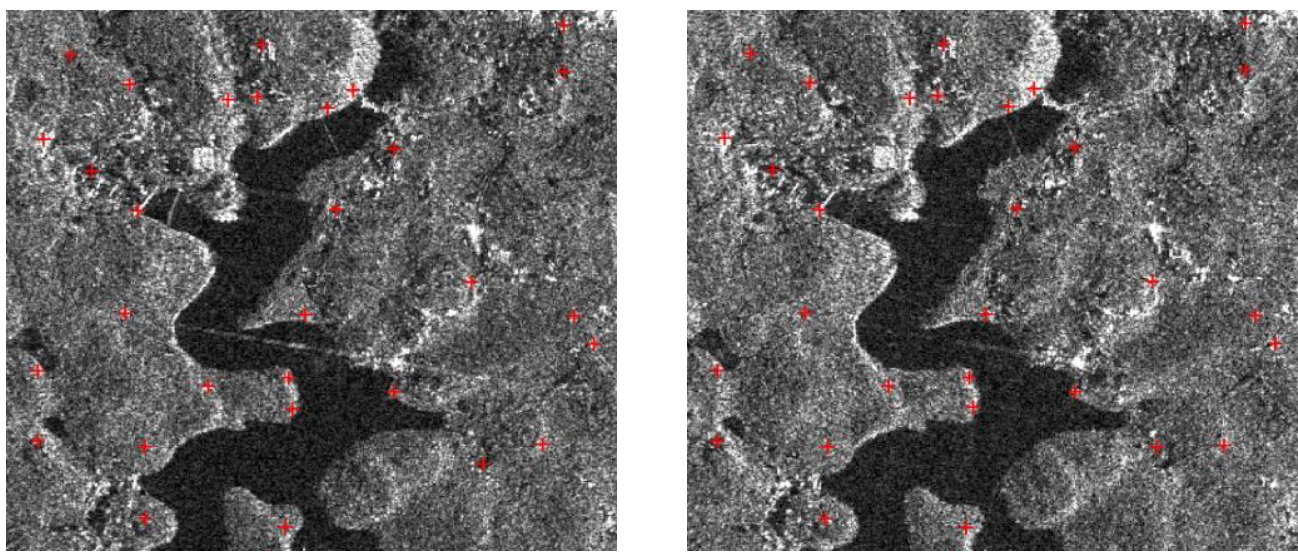

Figure5. Results of Test Image in Water Area

\subsection{Experiment analysis}

The experimental results (in table 2) show that, the SNCC algorithm can be used to accurately find the points with the same name of the two images. In this paper, 30 feature points by SURF from each image were selected for experiment and matching error within about one pixel, the matching accuracy can reach $90 \%$. SURF matching algorithm can extract a large number of feature points, but the success rate of matching is not high. That is, using RANSAC algorithm to eliminate false matching points, there will still be an error match. Compared to the SURF matching results, the accuracy increased about $40 \%$. In addition, this method can control the distribution of matching points, obtain even distribution of the same-name points, and at the same time, it can avoid extracting points in deformation areas and water areas that have no practical significance, and can meet the requirements of actual mapping. But, this method is a semiautomated matching method and take a longer time to match.

\section{CONCLUSION}

In this paper, we use SURF to extract the feature points of SAR image and then use SNCC to extract the same name points, matching accuracy is improved and can effectively avoid the effect of geometric distortion on SAR image matching. Effectively solve the problem of matching difficulty caused by geometric deformation. But, this method is a semi-automated matching method and take a longer time to match. Need to study to improve matching efficiency in the further.

\section{ACKNOWLEDGEMENTS (OPTIONAL)}

The work was supported in part by the CASM Fundamental research funds (No.7771715)

\section{REFERENCES}

H. Bay, T. Tuytelaars, and L. Van Gool, "SURF: Speeded up robust features", In ECCV, 2006,pp. 404-417

Teng Wang, Sigurjón Jónsson, and Ramon F. Hanssen Improved SAR Image Coregistration Using Pixel-Offset Series[J]. IEEE Geosci. Remote Sens. Lett., Vol. 11, no. 9, SEPTEMBER 2014.

WEI S, LAI S. Fast template matching based on normal-ized cross correlation with adaptive multilevel winner up-[J]. IEEE Transactions on Image Processing, 2008, 17 (11) : 22272235.
Baumberg, A. Reliable feature matching across widely separated views[C]. In Conference on Computer Vision and Pattern Recognition, Hilton Head, South Carolina, 2000:774781.

Herbert Bay, Andreas Ess, Tinne Tuytelaars, and Luc Van Gool,"Speeded-Up Robust Features (SURF)", Computer Vision and Image Understanding, v.110, n.3, June, 2008, pp.346-359

R.J.M.den Hollander,A. Hanjalic,“A six-point RANSAC algorithm for fundamental matrix estimation", Technical Report ICT, 2007.

Paillou P, Gelautz M. Relief reconstruction from SAR stereo pairs: the "optimal gradient" matching method[J]. IEEE Transactions on Geoscience and Remote Sensing,1999,37(4):2099-2107.

Capaldo P, Crespi M, Fratarcangeli F, et al. High-Resolution SAR radargrammetry: a first application with COSMO-SkyMed spotLight imagery[J]. IEEE Geoscience and Remote Sensing Letters, 2011,8(6):1100-1104.

CHEN Er-Xue, LI Zeng-Yuan, TIAN Xin, LI Shi-Ming , "The Application of Scale Invariant Feature Transformation to SAR Imagery Registration”. ACTA AUTOMATICA SINICA (in chinese), Vol.34, No.8,august, 2008,pp. 861-868.

ZITOVA B, FLUSSER J. Image registration methods : a survey [ J ] Image and Vision Computing, 2003，21（11）: 977-1000.

Revised January 2018 